\title{
Salicylic acid can reduce infection symptoms caused by Dickeya solani in tissue culture grown potato (Solanum tuberosum L.) plants
}

\author{
Robert Czajkowski • Jan M. van der Wolf • Aleksandra Krolicka • \\ Zofia Ozymko • Magdalena Narajczyk • Natalia Kaczynska • \\ Ewa Lojkowska
}

Accepted: 4 November 2014 / Published online: 13 November 2014

(C) The Author(s) 2014. This article is published with open access at Springerlink.com

\begin{abstract}
The potential for control of Dickeya solani infections in potato by elicitation of in vitro grown potato plants with salicylic acid (SA) was investigated by selective plating and confocal laser scanning macroscopy (CLSM). In replicated experiments potato plants grown on medium supplemented with 25 or $50 \mu \mathrm{M}$ of SA were evaluated for the phytotoxic effects. Potato plants grown on medium supplemented with SA and inoculated with GFP-tagged $D$. solani were investigated for blackleg development and colonization of potato plants by the bacteria. Three days after inoculation, colonization of roots by $D$. solani was observed in
\end{abstract}

R. Czajkowski $(\bowtie) \cdot$ Z. Ozymko • N. Kaczynska •

E. Lojkowska

Laboratory of Plant Protection and Biotechnology,

Department of Biotechnology, Intercollegiate Faculty of

Biotechnology, University of Gdansk and Medical University

of Gdansk, Kladki 24, 80-822 Gdansk, Poland

e-mail: robert.czajkowski@biotech.ug.edu.pl

J. M. van der Wolf

Plant Research International, P.O. Box 69, 6700

AB Wageningen, The Netherlands

A. Krolicka

Laboratory of Biologically Active Compounds, Department of Biotechnology, Intercollegiate Faculty of Biotechnology, University of Gdansk and Medical University of Gdansk,

Kladki 24, 80-822 Gdansk, Poland

M. Narajczyk

Laboratory of Electron Microscopy, Faculty of Biology,

University of Gdansk, Wita Stwosza 59, 80-308 Gdansk,

Poland
$100 \%$ control plants grown on medium without SA but not in plants grown on medium supplemented with $50 \mu \mathrm{M}$ SA. After 14 days, $100 \%$ of control plants showed severe disease symptoms, whereas plants grown on medium supplemented with $50 \mu \mathrm{M}$ SA and inoculated with bacteria did not express any symptoms. After 14 days bacteria were found inside $100 \%$ stems of control plants in densities of ca. $10^{3}-10^{4} \mathrm{cfu} \mathrm{g}^{-1}$ and inside $c a$. $10-15 \%$ stems of plants treated with $50 \mu \mathrm{M}$ SA in densities similar to these in the control plants. The GFP-tagged bacteria were macroscopically detected on the surface of the roots of control plants but not on the surface of the plants treated with $50 \mu \mathrm{M}$ SA 14 days after inoculation. The implications of SA treatments on plant fitness and disease development are discussed.

Keywords Blackleg Erwinia chrysanthemi .

Biological control · Induced resistance · Elicitation · Dose effect. SAR

\section{Introduction}

Bacterial diseases of potato, blackleg and soft rot, caused by pectinolytic Pectobacterium and Dickeya spp. are responsible for major losses in seed and ware potato production in Europe and worldwide (Pérombelon and Salmond 1995; Pérombelon 2002; Toth et al. 2011). In Europe, the Dickeya spp. outbreaks have increased substantially since 2001, when the isolates of the new Dickeya species (D. solani) have been 
found in potato for the first time (Slawiak et al. 2009ab). This pathogen has never before been observed in the European climate zone or elsewhere (van der Wolf et al. 2014; Tsror et al. 2009; Slawiak et al. 2009; Laurila et al. 2008). Since 2005 , the presence of $D$. solani has been reported in potato in many European countries including The Netherlands, Finland, Poland, Germany, Belgium, France, United Kingdom and Sweden, as well as outside Europe, in Israel and Georgia. The species has become the predominant pathogen responsible for the blackleg incidences in Europe (Toth et al. 2011; Tsror et al. 2011; van der Wolf et al. 2014). D. solani seems to be virulent in potato and possesses features allowing its easy spread and survival under the temperate climate in Europe (Czajkowski et al. 2009, 2012a).

The potential to control pectinolytic Dickeya spp. in potato is limited (Czajkowski et al. 2012b). Up to now, only an integrative strategy, combining the use of certified seed tubers descending from pathogen-free minitubers, hygienic practices during harvesting and grading, and avoidance of pathogen introduction during planting and in storage, is partially efficient but has not resulted in a broad eradication of the pathogens from the potato production chain (Perombelon and Kelman 1980; Toth et al. 2011).

Reduction of Pectobacterium and Dickeya spp. populations by treatment of potato tubers is ineffective and consequently rarely used in practical applications in fields (van der Wolf and de Boer 2007). Both, physical treatments including hot air and hot water, UV radiation and solarization, and utilization of chemical agents combining disinfectants and antibiotics, contribute to reduction or eradication of only superficial bacterial populations, whereas, they will not affect bacteria located deeper inside the tubers (Czajkowski et al. 2012b). Pectobacterium and Dickeya spp. are vascular pathogens, able to infect potato tubers internally (Perombelon and Kelman 1980; Czajkowski et al. 2009, 2010) and due to the ability of inhabiting the inner tuber tissues they will not be affected by the superficial treatments, thus the physical measures and chemical control agents will not effectively contribute to the control of Pectobacterium and Dickeya spp. in potato.

The use of elicitors, inducing natural resistance in plants against bacterial infections, can be considered as a promising alternative to chemical and physical treatments. It is well established that plants have evolved a range of different defence mechanisms to combat invasion and infections caused by bacterial pathogens.
Despite the presence of passive physical and chemical barriers, different mechanisms are also activated specifically upon pathogen introduction and infection (Kessmann et al. 1994). The induced resistance (IR) may be restricted to the site of the pathogen entrance, or it may spread systemically and develop also in other (healthy) distant parts of the plant (Durrant and Dong 2004).

The two best characterized defence mechanisms in plants are: induced systemic resistance (ISR) and systemic acquired resistance (SAR) (Vallad and Goodman 2004). Activation of the ISR and SAR leads to similar phenotypic responses, however, the molecular and biochemical pathways that are activated in these defence mechanisms are different. SAR is mainly induced by the exposure of roots or foliar tissues (leaves and haulms) to abiotic and biotic elicitors and depends on the salicylic acid signal molecule (Ryals et al. 1994). Activation of SAR leads to production and accumulation of specific pathogenesis-related proteins (PR proteins) (Métraux et al. 2002). In contrast, ISR is prompted by the presence of plant-growth promoting rhizosphere bacteria in plant rhizospheres and is mediated by the plant hormones, ethylene and jasmonic acid (Van Loon et al. 1998). Also, ISR is not associated with production of PR proteins (Heil and Bostock 2002).

Certain chemicals, e.g. salicylic acid (SA), benzothiadiazole (BTH), acybenzolar-S-methyl (BI$\mathrm{ON}), 2,6$-dichloroisonicotinic acid or $\beta$-aminobutyric acid (BABA) can activate the SAR defence mechanism without the plant's interaction with pathogens (Gozzo 2003). Whereas ISR most often requires the presence of living microorganisms or their fragments (e.g. pathogen proteins, pathogen cell walls components) for induction (Pieterse et al. 2001). ISR can also be enhanced by applying exogenous plant hormones: jasmonic acid and ethylene, or their derivatives (Shoresh et al. 2005; Pieterse et al. 2000). For example, Luzzatto et al. (2007a) demonstrated that calla lily (Zantedeschia spp.) plants exposed to exogenous BION or methyl jasmonate elicitors produced high levels of antimicrobial phenolics. It was also shown that elicitation of these plants with methyl jasmonate but not with BTH induces priming and consequently resistance against $P$. carotovorum via induction of oxido-reductases (Luzzatto-Knaan et al. 2014).

Although SA and its analogs have been extensively used in research on systemic resistance in different plants, relatively little information can be found on its 
role in protection against the pectinolytic bacteria. Palva et al. (1994) reported that addition of the SA to the growth medium of axenically-growing tobacco seedlings causes almost complete resistance to infection by P. carotovorum subsp. carotovorum and to our knowledge, there is no information available on the effect of SA on Dickeya spp., particularly the D. solani infections of potato.

The aim of this work was to investigate the effect of application of SA on symptom development caused by D. solani on in vitro grown potato plants. In replicated experiments the potato plants grown on medium supplemented with SA were investigated for the phytotoxic effects. In line with this, potato plants grown on a medium supplemented with SA and stem baseinoculated with GFP-tagged D. solani (Czajkowski et al. 2010) were investigated for the blackleg development, bacterial population dynamics in stems and colonization of plant roots by the GFP-tagged bacteria.

\section{Materials and methods}

Bacterial strains and media

GFP-tagged D. solani IPO2222 (IPO2254) (Czajkowski et al. 2010) was grown at $28^{\circ} \mathrm{C}$ for $24-48 \mathrm{~h}$ on tryptone soya agar (TSA) (Oxoid) or nutrient agar (NA) (Oxoid), supplemented with $150 \mu \mathrm{g} \mathrm{ml}^{-1}$ ampicillin (Sigma) prior to use. Liquid cultures were prepared in nutrient broth (NB) (Oxoid) and/or in tryptic soya broth (TSB), if required - also supplemented with ampicillin and grown at $28^{\circ} \mathrm{C}$ for $24 \mathrm{~h}$ with agitation $(200 \mathrm{rpm})$.

Salicylic acid (SA)

Salicylic acid (Sigma) was prepared as hundred or thousand times concentrated stock in $50 \%$ ethanol prior to use. The SA was added to the MS growth medium to the final concentration of 25 or $50 \mu \mathrm{M}$.

Assessment of D. solani IPO2254 morphology in transmission electron microscopy (TEM) upon treatment with SA

To assess the morphology of $D$. solani cells treated with $50 \mu \mathrm{M}$ SA, bacteria were grown overnight in TSB at $28^{\circ} \mathrm{C}$ with shaking $(200 \mathrm{rpm})$ in the presence of $50 \mu \mathrm{M}$ $\mathrm{SA}$. For the TEM analysis bacteria were adsorbed onto carbon-coated grids (Sigma) stained with $1.5 \%$ uranyl acetate and directly examined with electron microscope (Philips CM100, EM, FEI Company) as described earlier (Szpitter et al. 2014). The experiment was repeated independently one time with the same setup.

Measuring the antibacterial effect of salicylic acid

To check the possible direct effect of SA on $D$. solani growth in vitro, an overnight IPO2254 bacterial culture with a density of $c a .10^{9}$ cells ml $^{-1}$ in NB supplemented with $150 \mu \mathrm{g} \mathrm{ml}^{-1}$ ampicillin was diluted 50 times in the same medium supplemented with SA to the final concentration of $50 \mu \mathrm{M}$. The prepared bacterial cultures were grown at $28{ }^{\circ} \mathrm{C}$ with agitation $(200 \mathrm{rpm})$. For negative control, bacteria were prepared under the same conditions, without SA. Growth rate was determined by measuring the optical density $\left(\mathrm{OD}_{600}\right)$ for a period of $12 \mathrm{~h}$ each hour and one time after 24 and after $48 \mathrm{~h}$ and by comparing the growth of SA treated bacterial cultures with the growth of control cultures. Bacterial densities were estimated from the $\mathrm{OD}_{600}$ reads assuming that $\mathrm{OD}_{600}=0.1$ is equal to $10^{8} \mathrm{cfu} \mathrm{ml} \mathrm{m}^{-1}$. The experiment was repeated twice with the same set-up with two replicates for each experiment. The results were averaged.

Assessment of $D$. solani IPO2254 maceration ability on potato tuber slices upon treatment with SA

A potato slice assay (Czajkowski et al. 2010) was used to check if incubation of D. solani IPO2254 with SA prior to inoculation of potato tuber slices with the bacteria would affect its ability to macerate potato tuber tissue. For this, bacteria were grown for $12 \mathrm{~h}$ in TSB in the presence of $50 \mu \mathrm{M}$ SA with agitation $(200 \mathrm{rpm})$ at $28{ }^{\circ} \mathrm{C}$. After this time, bacteria were centrifuged for 5 min. at $6000 \times \mathrm{g}$ and washed two times with $1 / 4$ Ringer's buffer to remove the elicitor. Bacterial densities were adjusted to ca. $10^{6} \mathrm{cfu} \mathrm{ml}^{-1}$ with sterile demineralized water. For control, bacteria were grown in TSB under the same conditions as described above but without SA supplementation. Ware potato tubers of cv. Bryza (obtained at the local supermarket in Gdansk, Poland) were rinsed with running tap water, surfacesterilized with $70 \%$ ethanol for $10 \mathrm{~min}$, rinsed with running tap water and dried with tissue paper. Potato tubers were cut into ca. $0.7 \mathrm{~cm}$-thick transverse disks using a sterile table knife. Three wells $(5 \times 5 \times 5 \mathrm{~mm})$ per potato slice were made using a sterile cork borer and 
these were filled with $50 \mu \mathrm{l}$ of bacterial suspension containing $10^{6} \mathrm{cfu} \mathrm{ml}^{-1}$ in sterile demineralized water. For disease development slices were incubated at $28^{\circ} \mathrm{C}$ for $72 \mathrm{~h}$ in a humid box. Per treatment, three potato slices derived from three different potato tubers were used. For the negative control $50 \mu \mathrm{l}$ aliquots of sterile demineralized water were used instead of bacterial suspension. For the positive control, bacteria prepared in the same way but not treated with SA were used. The effect of SA on the ability of D. solani IPO2254 to macerate potato tuber tissue was measured by comparing the ratio of the average diameter of the rotting potato tissue around wells inoculated with SA-treated $D$. solani IPO2254 with the average diameter of rotting tissue around wells of the positive control (wells inoculated with $D$. solani IPO2254 not treated with SA prior to inoculation). Two repetitions were made per treatment and the entire experiment was independently repeated on time with the same set-up. Results from both experiments were averaged.

Growth and propagation of in vitro potato plants

In vitro potato plants cv. Kondor were obtained from the Laboratory of Seed Production and Potato Protection, Plant Breeding and Acclimatization Institute - National Research Institute, Bonin, Poland. Plants were grown on Murashige and Skoog (MS) medium (Murashige and Skoog 1962) with $20 \mathrm{~g} \mathrm{l}^{-1}$ sucrose and $7 \mathrm{~g} \mathrm{l}^{-1}$ agar. Potato plantlets were grown at a temperature of 20 $22{ }^{\circ} \mathrm{C}$ under white fluorescent light with a $16 \mathrm{~h}$ photoperiod (white cool fluorescent light, Philips, TLD $58 \mathrm{~W} /$ $84 \mathrm{o}, 30-35 \mu \mathrm{mol} \mathrm{m}{ }^{-2} \mathrm{~s}^{-1}$ ) for 4 weeks.

The experiments with SA were conducted on micropropagated plants in individual culture tubes (Duchefa Biochemie b.v) using single-node cuttings on MS medium containing 25 or $50 \mu \mathrm{M}$ SA with $10 \mathrm{~g} \mathrm{l}^{-1}$ sucrose and $7 \mathrm{~g} \mathrm{l}^{-1}$ agar, $\mathrm{pH}$ 5.8. The plantlets were grown at $20-22{ }^{\circ} \mathrm{C}$ for 20 days prior to inoculation with bacteria under the same conditions as described above. The control plants were micropropagated as described above, without SA.

Inoculation of in vitro-grown potato plants with D. solani IPO2254

For inoculation of potato plants, $10 \mu \mathrm{l}$ of bacterial suspension in water containing $10^{5} \mathrm{cfu}$ (colony forming units) $\mathrm{ml}^{-1}$ of $D$. solani IPO2254 was pipetted into the interspace between the stem base and MS medium of each individual plant. For negative control, instead of bacterial suspension, $10 \mu \mathrm{l}$ of sterile demineralized water was used. Inoculated plants were grown at a temperature of $20-22{ }^{\circ} \mathrm{C}$ under white fluorescent light with a $16 \mathrm{~h}$ photoperiod (white cool fluorescent light, Philips, TLD $\left.58 \mathrm{~W} / 84 \mathrm{o}, 30-35 \mu \mathrm{mol} \mathrm{m}{ }^{-2} \mathrm{~s}^{-1}\right)$. Six treatments were applied on potato plants: (treatment a) plants grown on MS medium and inoculated with water (negative control), (treatment $b$ ) plants grown on MS medium and inoculated with D. solani IPO2254 (positive control), (treatment $c$ ) plants grown on MS medium supplemented with $25 \mu \mathrm{M}$ SA and inoculated with water, (treatment $d$ ) plants grown on MS medium supplemented with $25 \mu \mathrm{M} \mathrm{SA}$ and inoculated with IPO2254, (treatment e) plants grown on MS medium supplemented with $50 \mu \mathrm{M}$ SA and inoculated with water and (treatment $f$ ) plants grown on MS medium supplemented with $50 \mu \mathrm{M}$ SA and inoculated with D. solani IPO2254. Per treatment, 20 plants grown in individual culture tubes were used and the entire experiment was repeated independently one time with the same set-up. Plants were visually inspected after 2, 5 and 10 days post inoculation (dpi) for symptom development and were sampled 14 dpi.

Symptom development in in vitro-grown potato plants

In vitro plants were sampled 14 dpi. Potato plants were assessed for wilting, typical blackleg, stem desiccation and plant death. The plants were aseptically removed from the in vitro plastic tubes and per plant, 1-cm long stem fragments taken $1 \mathrm{~cm}$ above the stem base were separately suspended in $1 \mathrm{ml}$ of 1/4-strength Ringer's buffer (Merck) supplemented with $0.02 \%$ diethyldithiocarbamic acid (Arcos Organics) in 2-ml Eppendorf tubes. Three sterile glass beads (Merck Chemicals) were added to each tube and the suspended stem fragments were vigorously vortexed ( $c a .300 \mathrm{rpm}$ ) for $60 \mathrm{~min}$ to grind plant tissues and release bacteria from plant fragments. One hundred $\mu$ of the undiluted and serial diluted stem extracts were plated in duplicates on CVP (Hélias et al. 2011) and/or TSA plates supplemented with ampicillin $\left(100 \mu \mathrm{g} \mathrm{ml}^{-1}\right)$ (Sigma). Plates were incubated for bacterial growth and cavity formation at $28^{\circ} \mathrm{C}$ for $24-48 \mathrm{~h}$. The resulting cavity forming, GFP positive, ampicillin resistant colonies were counted and the colony forming units per mg of stem tissue were calculated for each sample. 
Assessment of D. solani IPO2254 virulence on in vitro grown potato plants upon treatment with SA

To check the effect of SA on the virulence of D. solani IPO2254 on in vitro grown potato plants, bacteria were grown for $12 \mathrm{~h}$ in TSB supplemented with $50 \mu \mathrm{M}$ SA or in TSB at $28{ }^{\circ} \mathrm{C}$ with agitation (200 rpm). After this time, bacteria were centrifuged for $5 \mathrm{~min}$. at $6000 \times \mathrm{g}$ and washed two times with $1 / 4$ Ringer's buffer. Bacterial densities were adjusted to ca. $10^{5} \mathrm{cfu} \mathrm{ml}^{-1}$ with sterile demineralized water. In vitro potato plants of cultivar Kondor were propagated in a similar way as described above. The plantlets were grown at $20-22{ }^{\circ} \mathrm{C}$ for 20 days prior to inoculation with bacteria. For inoculation, $10 \mu \mathrm{l}$ of bacterial suspension in water containing $10^{5} \mathrm{cfu} \mathrm{ml}^{-1}$ of D. solani IPO2254 or $10^{5} \mathrm{cfu} \mathrm{ml}^{-1}$ of D. solani IPO2254 pretreated with SA were pipetted into the interspace between the stem base and MS medium of each individual plant. For negative control, instead of bacterial suspension, $10 \mu \mathrm{l}$ of sterile demineralized water was used. Inoculated plants were grown at a temperature of $20-22{ }^{\circ} \mathrm{C}$ under white fluorescent light with a $16 \mathrm{~h}$ photoperiod (white cool fluorescent light, Philips, TLD $58 \mathrm{~W} / 84 \mathrm{o}, 30-35 \mu \mathrm{mol} \mathrm{m}{ }^{-2} \mathrm{~s}^{-1}$ ) as described above. In vitro plants were sampled 14 dpi. Potato plants were visually assessed for wilting, typical blackleg, stem desiccation and plant death and the percentage of blackleg-diseased plants was calculated per each treatment. Per treatment 10 individual plants were used and the experiment was repeated one time with the same setup. Per treatment and per experiment, five randomly chosen stem fragments with the length of $1 \mathrm{~cm}$ collected $1 \mathrm{~cm}$ above the MS medium were assessed for D. solani IPO2254 densities in stems by counting serially diluted in Ringer's buffer GFP-positive ampicillin resistant colonies on TSA as described above.

Presence of $D$. solani strain IPO2254 on the surface of potato roots and stem bases assessed with confocal laser scanning macroscope

To check the presence of $D$. solani IPO2254 on roots and stem bases of inoculated plants, at $14 \mathrm{dpi}$, four individual plants of each treatment (in total 16 plants per experiment: four plants grown on MS medium and inoculated with sterile water (treatment a), four plants grown in MS medium and inoculated with
D. solani IPO2254 (treatment b), four plants grown in MS supplemented with $25 \mu \mathrm{M}$ SA and inoculated with IPO2254 (treatment $d$ ), and four plants grown in MS supplemented with $50 \mu \mathrm{M} \mathrm{SA}$ and inoculated with IPO2254 (treatment f)) were removed from the culture tubes, washed briefly in sterile water and examined for the presence of a GFP signal using a confocal Leica TCS LSI macro confocal microscope (Leica) at magnification $1 \times$ and $5 \times$. For this, plants were removed from culture tubes, placed on square transparent glass slides and examined directly with a macroscope without any further processing or incubation. Photographs were taken with a Leica Digital System (Leica) connected to the macroscope. The plants grown on MS medium supplemented with 25 or $50 \mu \mathrm{M}$ SA but not inoculated with bacteria (treatment $c$ and treatment $e$ ) were not examined with the macroscope.

\section{Statistical analysis}

Bacterial count data were analyzed with ordinary linear regression using the statistical software package Statistica ver. 10 (Statsoft, http://www.statsoft.com/). To achieve approximate normality, the data were $\log$ transformed after adding a value 1 to avoid taking $\operatorname{logs}$ of zero. Results were considered to be significant at $P=$ 0.05 and pair-wise differences were obtained using the $t$ test. Data were analyzed according to the experimental design e.g. two replicated in vitro experiments with treatments of 20 replications (plants) each. The linear model considered was a complete block design with replicates as complete block.

\section{Results}

Antibacterial effect of salicylic acid

No difference in D. solani cell morphology was observed in bacterial cultures grown in TSB with and without SA supplementation in transmission electron microscopy analysis (Fig. 1a). Treatment of D. solani IPO2254 with salicylic acid in concentration of $50 \mu \mathrm{M}$ for a period of $12 \mathrm{~h}$ measured every hour and one time after 24 and $48 \mathrm{~h}$ did not affect significantly the bacterial growth in comparison with control as determined by measuring the $\mathrm{OD}_{600}$ (Fig. 1b). 
Effect of salicylic acid on D. solani IPO2254 virulence

The ability of SA-treated D. solani IPO2254 to macerate potato tuber tissue was investigated using a potato slice assay. After incubation of slices for 3 days at $28{ }^{\circ} \mathrm{C}$, the diameter of the rotting tissues caused by the SA-treated bacteria was not significantly different from that of the non-treated with SA D. solani IPO2254 (Fig. 2a).

The ability of SA-treated D. solani IPO2254 to infect potato plantlets was compared with the ability of non-treated with SA D. solani IPO2254 to establish an infection in potato plants and was investigated using the in vitro potato plants of cv. Kondor. Plants infected with SA-treated D. solani IPO2254 displayed similar infection characteristics as plants infected with non-treated $D$. solani IPO2254, indicating that the SA-treated bacteria were able to colonize and cause infection of in vitro grown potato plants and that their virulence was not affected by incubation with $50 \mu \mathrm{M} \mathrm{SA}$ for $12 \mathrm{~h}$ at $28^{\circ} \mathrm{C}$ prior to infection studies (Fig. 2b). Bacterial densities in stems infected with SA-treated and SA-untreated D. solani IPO2254 were not statistically different and on average densities of $10^{3}-10^{4} \mathrm{cfu} \mathrm{g}^{-1}$ of stems were found (data not shown).

Growth chamber experiments with in vitro grown potato plants

\section{Fitness of potato plants and disease development}

Experiments with in vitro plants were conducted in December 2013 - January 2014 (experiment 1) and January - February 2014 (experiment 2). In each experiment the effect of 2 concentrations of SA (25 and $50 \mu \mathrm{M}$ ) on plant fitness and disease progression caused by $D$. solani IPO2254 in inoculated plants $(n=20)$ was investigated (Fig. 3).

Treatment (a) In plants grown on MS medium and inoculated with sterile water, no disease symptoms were observed during the entire course of both experiments. In experiment 1 , the average height of plants at $14 \mathrm{dpi}$ was $4.8 \mathrm{~cm}$ and the average fresh weight of haulms was $61.7 \mathrm{mg}$. In experiment 2 the average height of plants was $5.5 \mathrm{~cm}$ and the average fresh weight was $62.4 \mathrm{mg}$.
Fig. 1 Effect of $50 \mu \mathrm{M}$ salicylic acid (SA) on the morphology (A) and growth (B) of $D$. solani IPO2254 in vitro. a To asses morphology of bacterial cells upon treatment with SA transmission electron microscopy (TEM) analysis was conducted on cells of D. solani IPO2254 grown overnight in Tryptone Soya Broth supplemented with $50 \mu \mathrm{M}$ SA with shaking $(200 \mathrm{rpm})$ at $28^{\circ} \mathrm{C}$ and for control in TSB without SA supplementation. Photos were taken directly after bacteria collection from liquid cultures. For this, bacteria were adsorbed onto carbon-coated grids (Sigma) stained with $1.5 \%$ uranyl acetate and directly examined with electron microscope (Philips CM100, EM, FEI Company). The experiment was repeated independently one time with the same setup. The figure shows representative cells. b To assess the growth of bacterial cells upon treatment with $\mathrm{SA}$ the growth rate of D. solani IPO2254 cells in TSB and TSB supplemented with $50 \mu \mathrm{M}$ SA was determined by measuring the optical density (OD600) for a period of $12 \mathrm{~h}$ each hour and one time after 24 and 48 hours. Bacterial densities were estimated from the $\mathrm{OD}_{600}$ reads assuming that $\mathrm{OD}_{600}=0.1$ is equal to $10^{8} \mathrm{cfu} \mathrm{ml}^{-1}$. The experiment was repeated twice with the same setup with two replicates for each experiment

Treatment (b) In plants grown on MS medium and inoculated with $D$. solani IPO2254, the first symptoms appeared 3 days after inoculation in both experiments. These were: colonization of roots observed as an increase of turbidity of MS medium around the roots and induction of stem base rotting and blackening progressing up the stems. In both experiments, after 14 days all plants showed typical blackleg symptoms. The infections resulted in a severe reduction of the plant height of $40 \%$ percent in experiment 1 and $49 \%$ in experiment 2, respectively, and of the fresh weight with $54 \%$ reduction in experiment 1 and $52 \%$ in experiment 2 , respectively.

Treatment (c) In plants grown on MS medium supplemented with $25 \mu \mathrm{M}$ SA and inoculated with water, no disease symptoms were observed during the entire course of both experiments. The treatment with SA did not significantly affect plant height and fresh weight.

Treatment (d) In plants grown on MS medium supplemented with $25 \mu \mathrm{M}$ SA and inoculated with IPO2254 bacteria, the first symptoms appeared 3 days after inoculation, similarly as in Treatment (b). After 3 days, bacteria were present in the rhizosphere which was visualized as an increase of turbidity of the MS medium around plant roots. In the next 2-3 days, decay of the stem base was initiated which 
a

\section{IPO2254 in TSB}
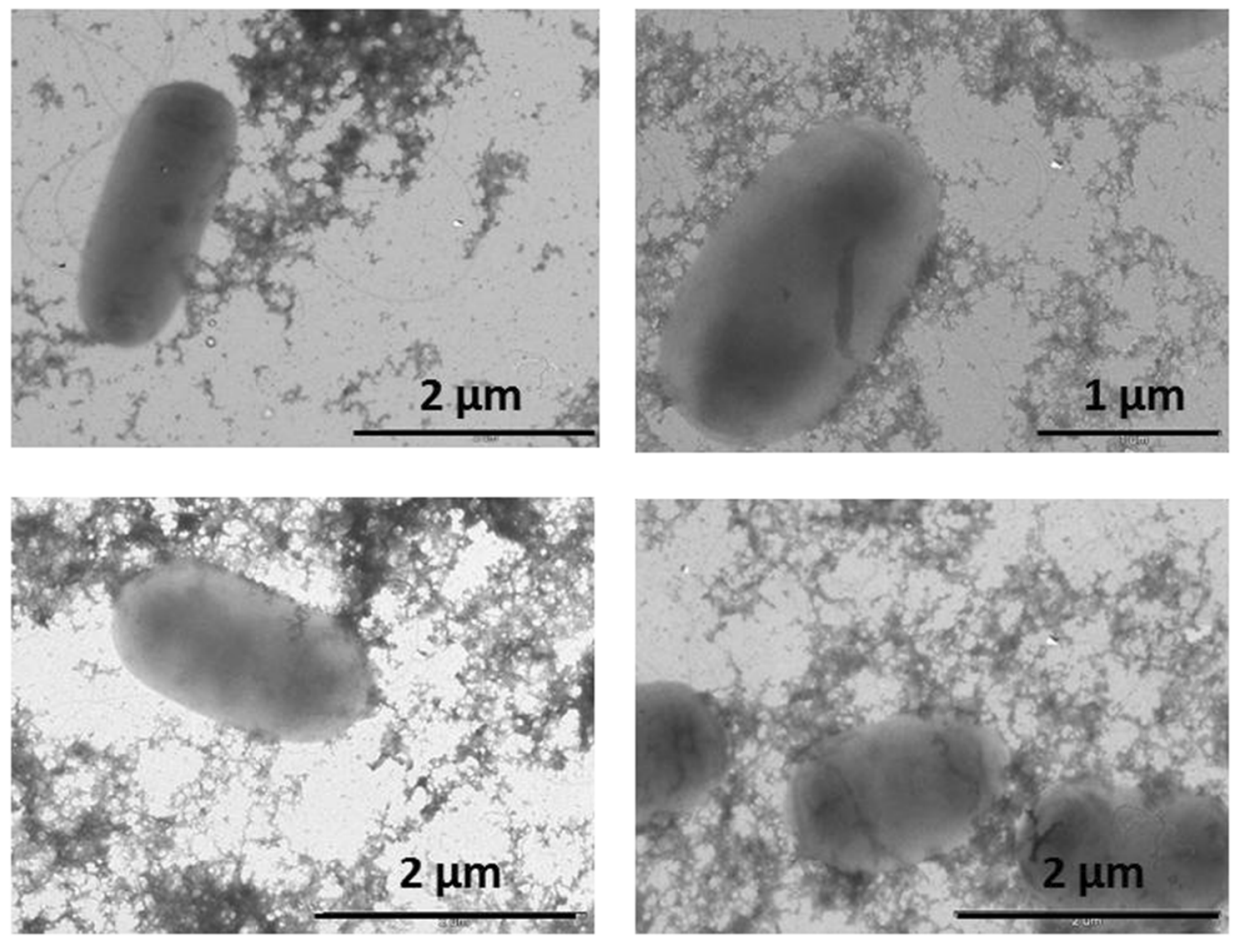

IPO2254 in TSB + 50 $\mu \mathrm{M}$ SA

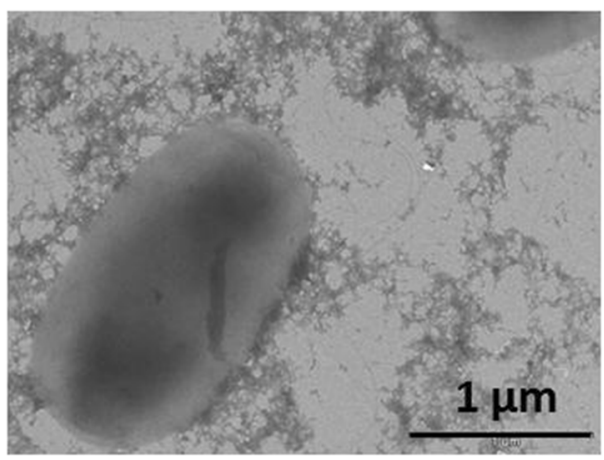

b 9

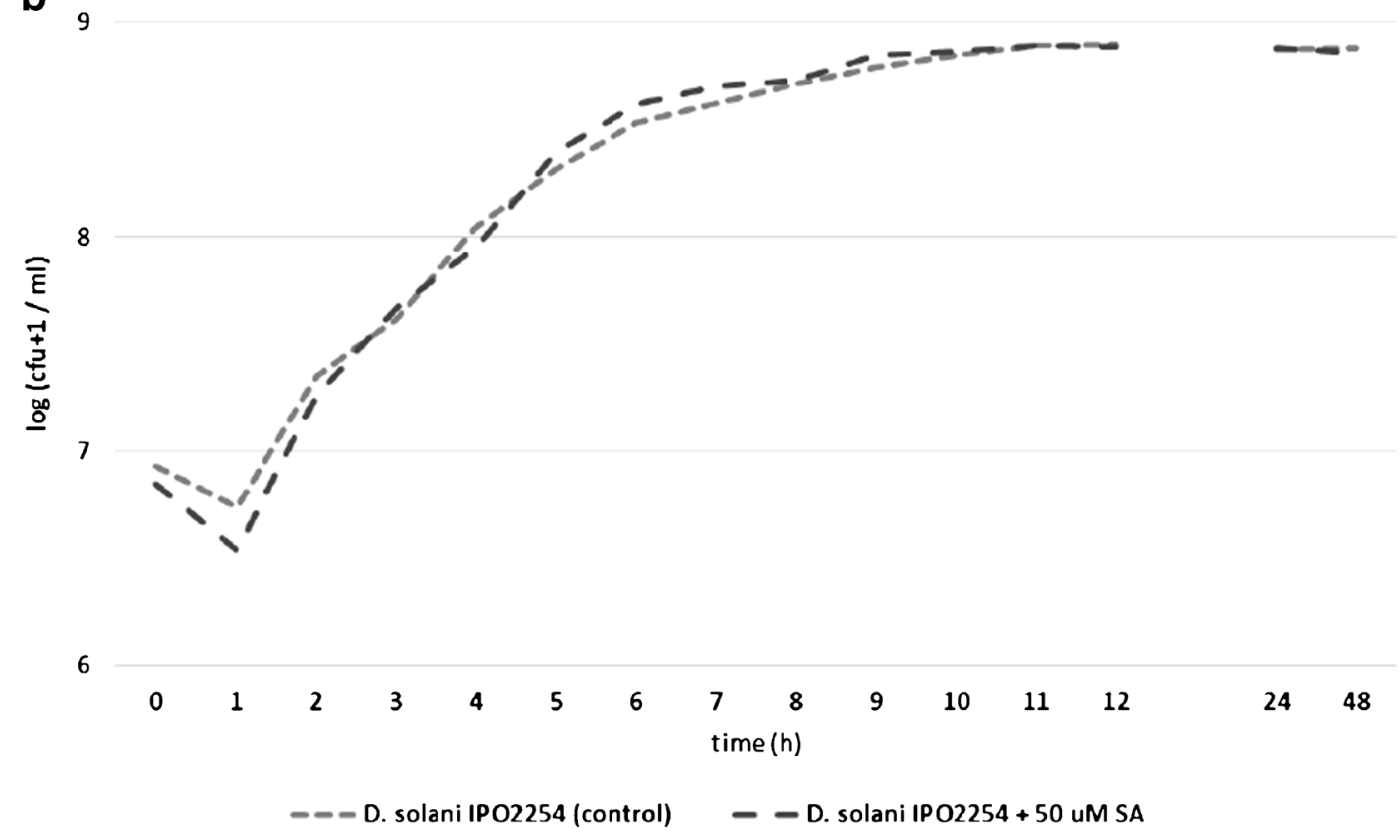

gradually extended along the stem. Due to severe infections after 14 days, blackleg symptoms were observed in $85 \%$ of plants in experiment 1 and in $80 \%$ 


\section{a}

50

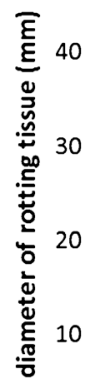

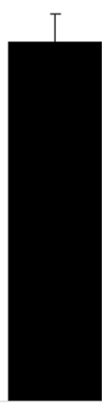

IPO2254

treatment

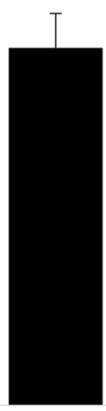

SA-treated IPO2254 water

b

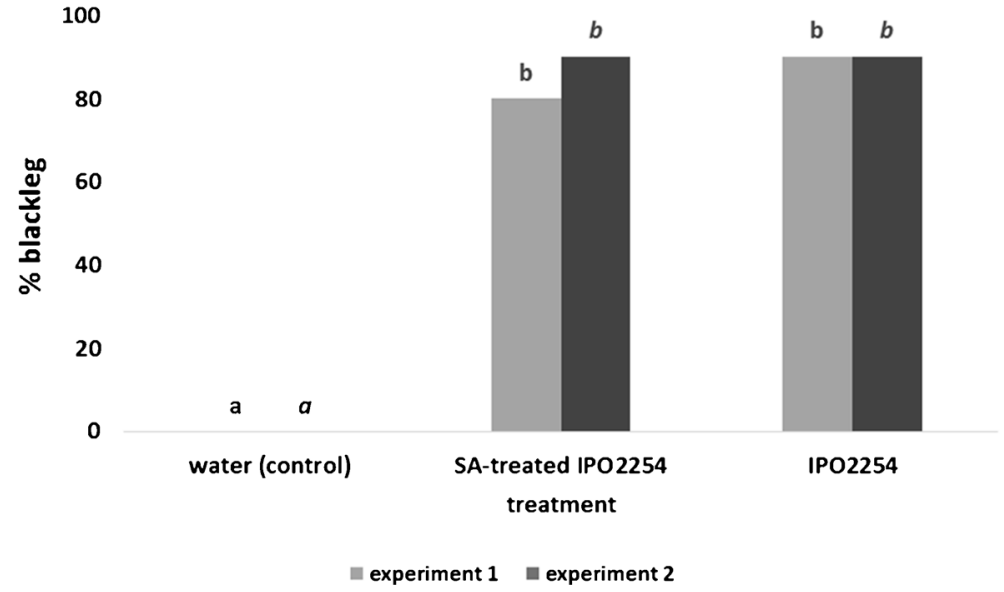

Fig. 2 The effect of $50 \mu \mathrm{M}$ SA on the $D$. solani IPO2254 ability to macerate potato tuber tissue (a) and the ability to cause blackleg symptoms in vitro grown potato plants (b). a To assess the ability of $D$. solani IPO2254 to macerate potato tuber tissue a potato slice assay was used. The diameter of rotting tissue (in $\mathrm{mm}$ ) of potato slices was measured after $72 \mathrm{~h}$ incubation at $28^{\circ} \mathrm{C}$ in a humid box. Wells of potato slices were filled up with $50 \mu$ of sterile water, with $50 \mu \mathrm{l}$ of bacterial suspension in water containing $10^{6} \mathrm{cfu} \mathrm{ml}^{-1}$ of $D$. solani IPO2254 or with $50 \mu \mathrm{l}$ of bacterial suspension in water containing $10^{6} \mathrm{cfu} \mathrm{ml}^{-1}$ of h $D$. solani IPO2254 pretreated with $50 \mu \mathrm{M}$ SA for $12 \mathrm{~h}$ and washed two times in Ringer's buffer prior to potato slice assay. Three potato slices containing 3 wells each and derived from three different tubers were used per

with IPO2254. Similarly, the shoot length was $76 \%$ and $73 \%$ of the shoots of control plants in experiment 1 and 2 , respectively.

Treatment (e) In plants grown on MS medium supplemented with $50 \mu \mathrm{M} \mathrm{SA}$ and inoculated with water, no disease or other symptoms were observed in both experiments. No statistically significant reduction of shoot treatment. The experiment was independently repeated one time and the results were averaged. $\mathbf{b}$ To assess the ability of $D$. solani IPO2254 to cause blackleg symptoms, in vitro grown potato plants of cv. Kondor were inoculated with $10 \mu \mathrm{l}$ of $D$. solani IPO2254 suspension in water containing $10^{5} \mathrm{cfu} \mathrm{ml}^{-1}$ or with $10 \mu \mathrm{l}$ of D. solani IPO2254 suspension pretreated with SA for $12 \mathrm{~h}$ and containing the same inoculum. For negative control, instead of bacterial suspension, $10 \mu \mathrm{l}$ of sterile demineralized water was used. Inoculated plants were grown at a temperature of $20-22{ }^{\circ} \mathrm{C}$ under the same conditions as described above. At 14 dpi potato plants were visually assessed for blackleg symptoms and plant death. Ten plants were used per treatment and the entire experiment was independently repeated one time with the same setup

length and fresh plant weight was observed in both experiments.

Treatment (f) In plants grown on MS medium supplemented with $50 \mu \mathrm{M} \mathrm{SA}$ and inoculated with $D$. solani IPO2254, no blackleg symptoms were observed in both experiments. Three days post inoculation in $10 \%$ plants and $15 \%$ plants (two plants in experiment 1 and three 


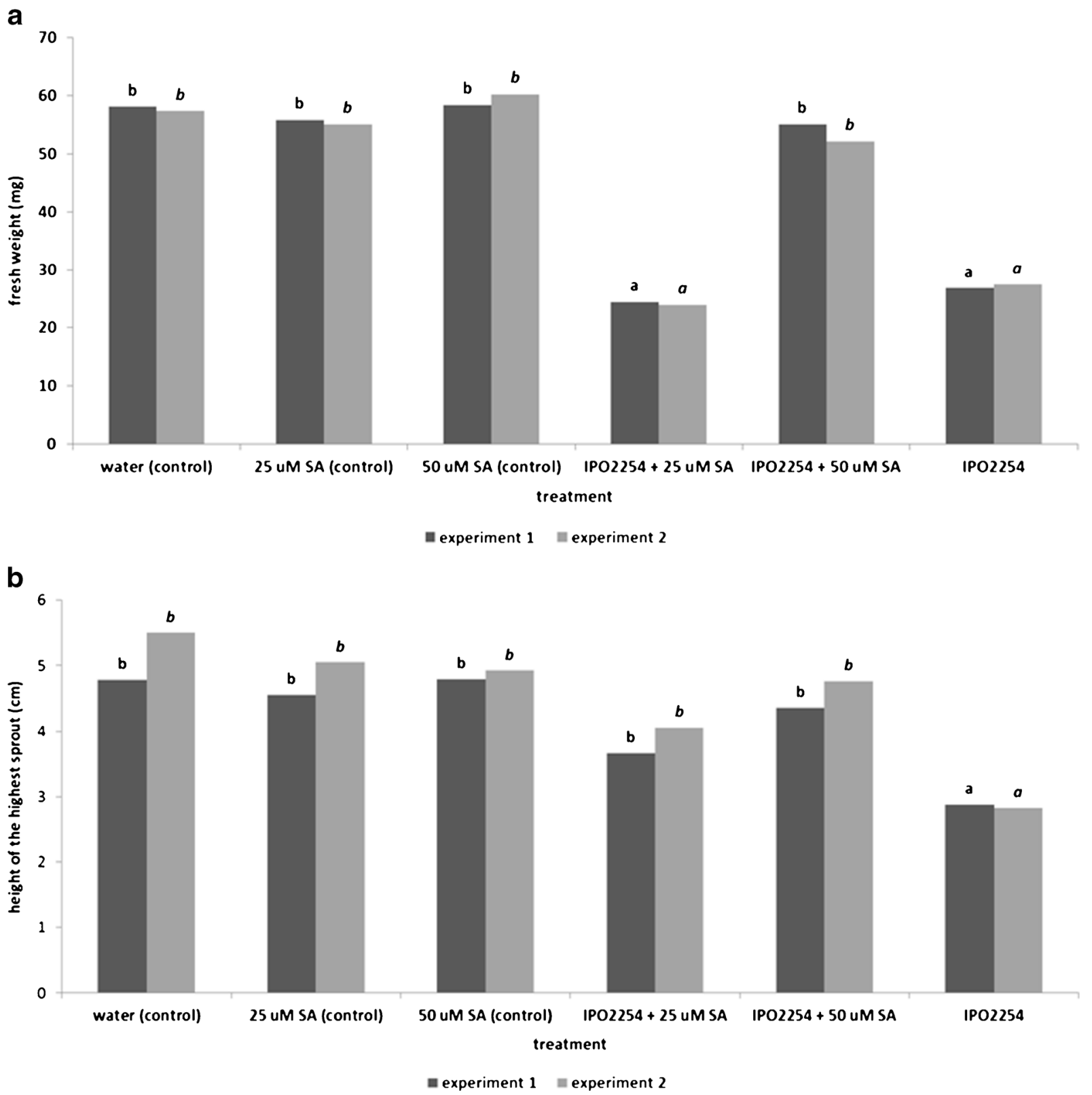

Fig. 3 Effect of SA and Dickeya solani IPO2254 on the fresh weight (a) and maximum shoot length (b) of axenically-grown potato plants. Plants were grown for 14 days on MS medium with or without 25 or $50 \mu \mathrm{M}$ SA prior to inoculation with the pathogen. Plants were analyzed after next 14 days (after 28 days in total) post or without inoculation with D. solani IPO2254. a Average fresh weight of plants determined 14 days post plant inoculation with bacteria. Control plants were inoculated with sterile water. Data from two independent experiments were analyzed separately. Values followed by identical characters are not significantly different $(P=0.05)$. b Average height of the highest shoot measured 14 days post plant inoculation with bacteria. Control plants were inoculated with sterile water. Data from two independent experiments were analyzed separately. Values followed by identical characters are not significantly different $(P=0.05)$ plants in experiment 2) colonization of roots by D. solani was observed. This, however, did not result in development of symptoms during the following
11 days (14 dpi). At the same time, only very low bacterial densities were found in the colonized plants (ca. 1-10 $\mathrm{cfu} \mathrm{g}^{-1}$ ) in both experiments. 


\section{Bacterial populations in stems}

Fourteen days after inoculation, stem fragments of in vitro potato plants were extracted and plated on TSA and/or CVP supplemented with ampicillin to determine the percentage of infected stems and to quantify internal populations of $D$. solani IPO2254. In both experiments no bacteria were found in the plants grown on MS medium supplemented with SA and inoculated with water (treatment a, treatment $c$ and treatment $e$ ) (Fig. 4a). Simultaneously, the GFP tagged strain was found in all stem fragments collected from plants grown on MS medium and inoculated with $D$. solani IPO2254 (treatment $b$ ), and in all stem fragments collected from plants grown on MS medium supplemented with $25 \mu \mathrm{M}$ SA and inoculated with D. solani (treatment $d$ ). In experiment 1 and $2,10 \%$ and $15 \%$ stem fragments, respectively, collected from plants grown on MS medium supplemented with $50 \mu \mathrm{M}$ SA and inoculated with strain IPO2254 (treatment $f$ ) harboured GFP-tagged bacteria. Bacterial densities in stems varied largely per plant in treatment $b$, treatment $d$ and treatment $f$, and on average the densities of $10^{2}-10^{4} \mathrm{cfu}^{-1}$ of $D$. solani were recorded (Fig. 4b).

\section{Confocal laser scanning macroscopy analysis}

Plant parts were analyzed with a Leica TCS LSI confocal macroscope (Leica) at a low magnification of 1 and 5 times. At 14 dpi, bright GFP signal was found on all roots and stem bases of sampled plants derived from treatment $b$ (plants grown on MS medium and inoculated with $D$. solani IPO2254) and in plants of treatment d (plants grown on MS medium with $25 \mu \mathrm{M} \mathrm{SA}$ and inoculated with IPO2254). No GFP signal was observed in the stem bases and roots of treatment $a$ (plants grown on MS medium and inoculated with sterile water) and in stem bases and roots of plants in treatment $f$ (grown on MS supplemented with $50 \mu \mathrm{M}$ SA and inoculated with bacteria) (Fig. 5).

\section{Discussion}

This study was conducted to assess the potential of SA as an elicitor to induce resistance against $D$. solani in potato. It is the first case dealing with SA treatment used in in vitro potato plants against infection caused by $D$. solani and also, the first description of a simple screening system combining in vitro (potato) plants, the fluorescently-labelled pathogenic bacteria and (fluorescent) macroscopy, that can be used on large number of (potato) plants and with different chemicals tested as prospective elicitors in vitro.

Elicitation of potato plants against pectinolytic bacteria has been analyzed previously in several studies (Palva et al. 1994; Vidal et al. 1997; Luzzatto et al. 2007b). For example, Palva et al. (1994) showed that the SA treatment together with application of P. carotovorum subsp. carotovorum on tobacco resulted in hypersensitivity reaction and induction of yetunknown factors able to neutralize the plant cell wall degrading enzymes produced by the pathogen. Vidal et al. (1997) demonstrated that compounds from P. carotovorum subsp. carotovorum may act antagonistically with SA elicitation. SA was observed to inhibit the induction of genes coding for pathogenies-related (PR) proteins elicited by the bacteria, which suggested a cross-talk of SA and bacterial elicitor network. To our best knowledge, there is no information available on elicitation of plants against Dickeya spp. and specifically against $D$. solani.

Addition of $50 \mu \mathrm{M}$ SA to the MS medium resulted in strong protection of plants against colonization of and infection with $D$. solani via developing roots. Plants treated with $50 \mu \mathrm{M}$ SA were symptomless whereas $100 \%$ of untreated plants showed symptoms. This suggests that the application of SA prevents bacteria from colonizing the root surface and systemic infections of potato plants. Similar observations on SA concentration in plants were reported for rice (Oryza sativa) and pea (Pisum sativum) where it was shown that application of exogenous SA may protect roots from being invaded by pathogens and/or from developing symptoms even up to $100 \%$ (Silverman et al. 1995; Blilou et al. 1999).

The efficacy of SA-mediated resistance of in vitro grown potato plants against $D$. solani appears to be dose dependent. At a concentration of $25 \mu \mathrm{M}$ SA the level of protection was only minor. In contrast, in a twice as high concentration $(50 \mu \mathrm{M})$ the protection effect was almost complete, and no blackleg symptoms in tested plants with IPO2254 were observed 14 dpi. Supplementation of 25 or $50 \mu \mathrm{M}$ SA to MS medium had no adverse effect on plant growth. At higher concentrations, i.e. 150 and $300 \mu \mathrm{M}$, salicylic acid caused deterioration of plant growth and abnormalities during leaf (e.g. yellowing and wilting) and root development (e.g. poor rooting) 

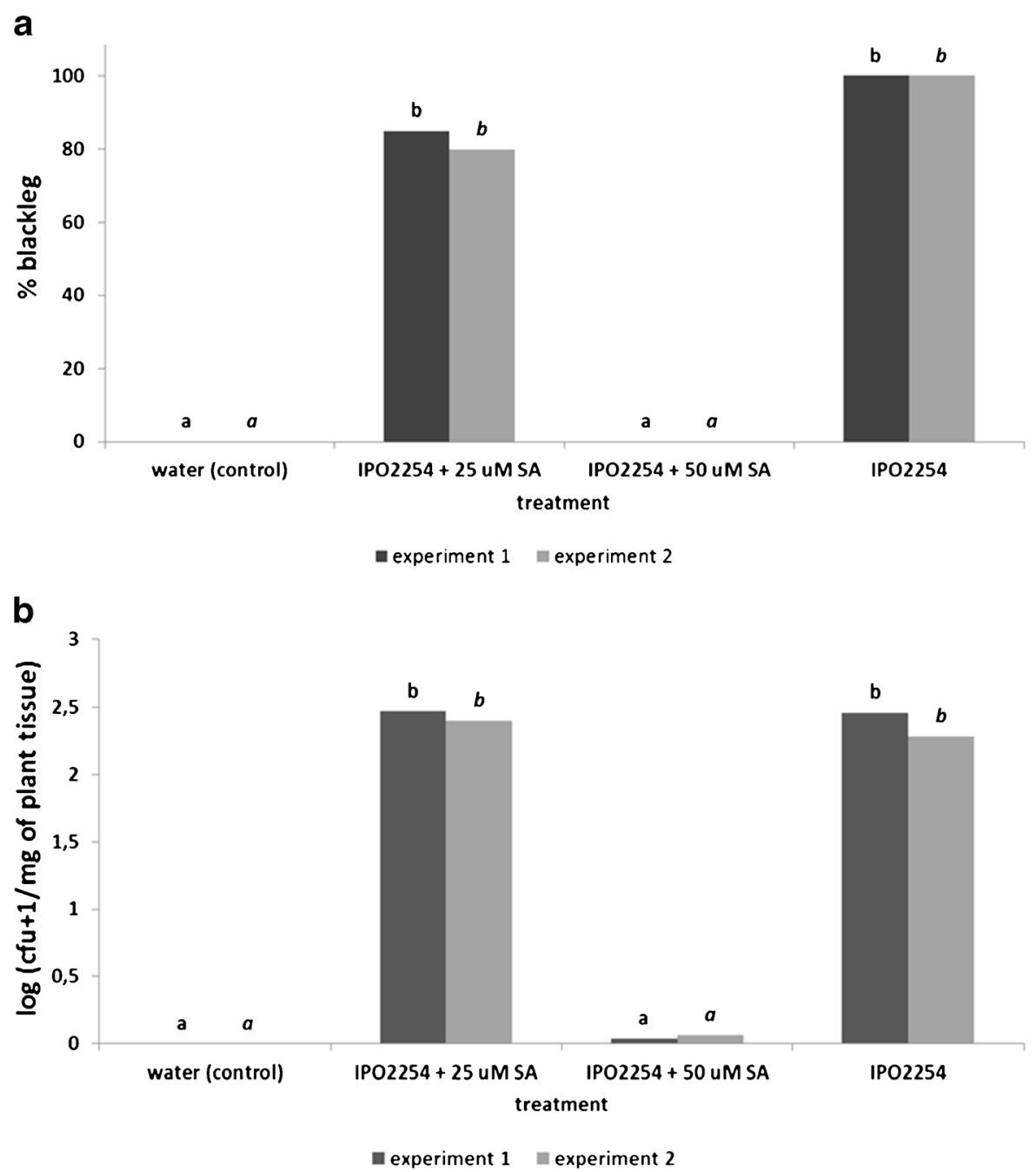

Fig. 4 Effect of supplementation of the growth medium with SA on the average percentage of symptomatic plants (showing blackleg or browning and rotting of stem tissue) 14 days post inoculation with bacteria ( $n=20$ plants per treatment) (a) and on GFP- tagged D. solani IPO2254 bacterial densities in stems $(n=20$ plants per treatment) (b). Values followed by identical characters are not significantly different $(P=0.05)$

serve as a method-of-choice for the first screening of other compounds for protection of (potato) plants against soft rot bacterial pathogens. The screening method can be scaled up easily if necessary.

The use of in vitro plants of cv. Kondor, highly susceptible to infections with Dickeya spp., resulted in a rapid colonization of plants. The use of the GFPtagged D. solani strain IPO2254 allowed microscopical observations of bacteria on root and stem base surface as it was described earlier by Golan et al. (2010), who used a GFP-tagged P. carotovorum subsp. carotovorum to follow the fate and population dynamics of bacterial cells upon infection of in vitro grown Ornithogalum 


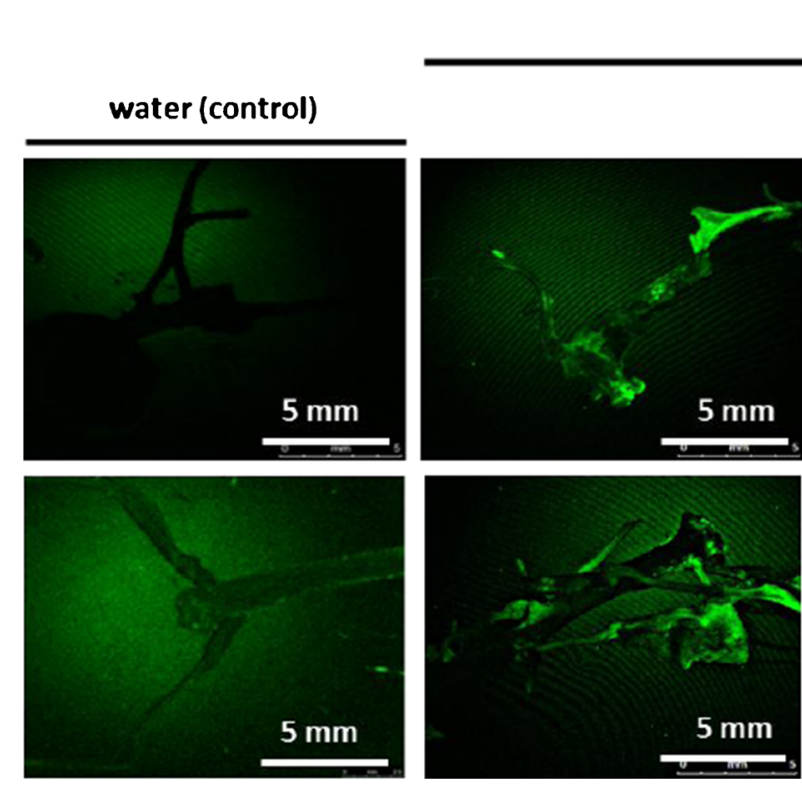

Fig. 5 Colonization of roots of in vitro grown potato plants with GFP-tagged D. solani IPO2254 assessed with confocal laser scanning macroscopy (CLSM). Potato roots were collected 14 days post inoculation and were briefly washed from MS medium with tap water and used immediately for macroscopic analysis. Samples were taken from plants inoculated with $D$. solani IPO2254, plants grown on MS medium supplemented with $25 \mu \mathrm{M}$ SA and inoculated with $D$. solani IPO2254, and plants grown on MS medium

dubium plants. This study shows that $D$. solani is able to enter roots from the medium whereupon the potato plants are systemically colonized as it was reported previously for plants grown under greenhouse conditions in soil (Czajkowski et al. 2010).

It has been reported previously that SA can cause morphological and transcriptional changes in bacterial cells yet this effect was evaluated mainly for human pathogens such as Serratia marcescens, Staphylococcus aureus and Klebsiella pneumoniae (Price et al. 2000; Delaney et al. 1994). Consequently, only very little information is present on its effect on virulence of plant pathogenic bacteria (Raskin 1992). We have found only one study describing the SA effect on P. carotovorum species close related to Dickeya spp. (Lagonenko et al. 2013). Lagonenko et al. (2013) showed that 25 and $50 \mathrm{mM}$ SA $(1000 \times$ times higher concentration than used in our study) negatively influenced important virulence determinants $i$. e. biofilm formation, motility and quorum sensing mechanism in $P$. carotovorum. However, it is likely that in our studies the effect of SA was exclusively plant-mediated as no direct antibacterial effect of $50 \mu \mathrm{M}$ on bacterial growth was observed. The IPO2254

\section{IPO 2254}
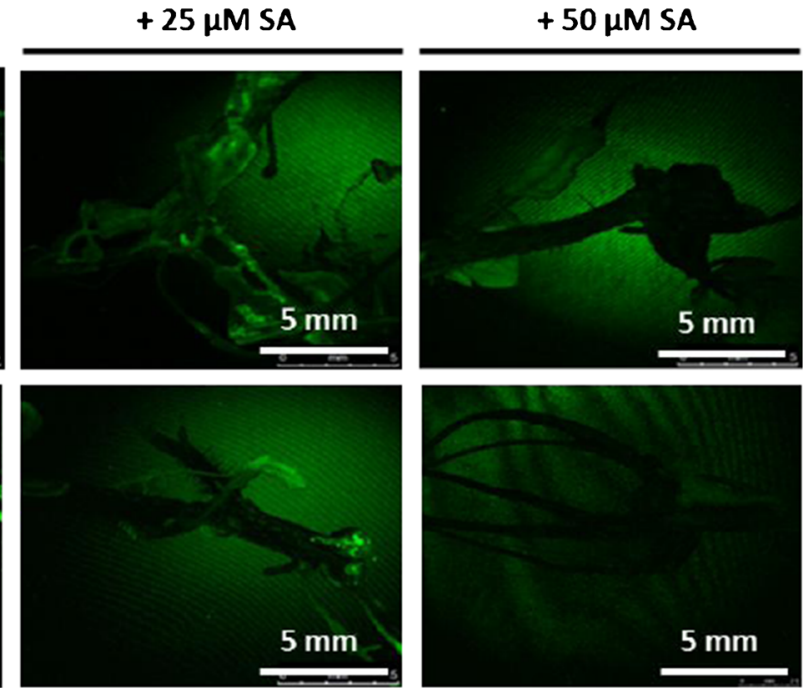

supplemented with $50 \mu \mathrm{M}$ SA and inoculated with $D$. solani IPO2254. For control, potato plants grown on MS medium were inoculated with sterile water. Green fluorescence was observed on roots and stem bases taken from plants inoculated with IPO2254 and in plants grown on medium supplemented with $25 \mu \mathrm{M} \mathrm{SA}$ and inoculated with IPO2254. No fluorescence was observed in plants grown on medium supplemented with $50 \mu \mathrm{M}$ SA and inoculated with bacteria

growth in liquid cultures containing the elicitor in the given concentration was similar to the growth of this strain in cultures without SA as evidenced by measuring the optical density of bacterial cultures up to $12 \mathrm{~h}$ and after 24 and $48 \mathrm{~h}$. Also, in the transmission electron microscopy analysis no effect of SA on the morphology of $D$. solani was observed. In line, we have not observed any negative effects of SA on bacterial virulence and ability to macerate potato tuber tissue in comparison with the untreated with SA D. solani IPO2254 using two different approaches - potato slice assays and in vitro grown potato plants. It cannot be excluded however, that higher concentrations of SA may cause morphological and physiological alternations in Dickeya spp. and particularly in D. solani, however at the concentration used in our study $(50 \mu \mathrm{M} \mathrm{SA})$ such effects were not observed. The protection of potato plants against $D$. solani by SA is therefore probably based on induced resistance of the treated plants, as it was reported earlier for other pathogens (Durrant and Dong 2004).

For a feasible application of SA in the control of potato blackleg caused by $D$. solani a considerable 
amount of work still needs to be done. There is a demand for information concerning various factors that may affect the influence of SA, including the time of application, the effect of the plant cultivar, the response against other pathogens causing blackleg and the most importantly, the impact of environmental conditions that would influence the expression of induced resistance in the field (Angelova et al. 2006). There is also a need to understand the mechanism of the protection afforded by SA to potatoes against $D$. solani. Work involving mass spectrometry analysis of potato-derived compounds elicited upon SA treatment and IPO2254 infection and experiments with hydroponically grown potato plants are now being conducted to understand the molecular basis of the SA-mediated elicitation of potato plants against $D$. solani and to find the time in which the elicitation effect last for, respectively.

Acknowledgments The work was financially supported by the Ministry of Science and Higher Education, Poland via a research grant Iuventus Plus 2012 (MNiSW 0241/IP/2013/72) to Robert Czajkowski and by the Polish Ministry of Science and Higher Education via statutory activity funds for 2013 to Aleksandra Krolicka. The authors would like to thank H. Rietman (Plant Research International, Wageningen, the Netherlands) for guidance in setup of the in vitro potato cultivation system, M. Obuchowski (Department of Molecular Bacteriology, Intercollegiate Faculty of Biotechnology, University of Gdansk and Medical University of Gdansk, Poland) for assistance in confocal laser scanning macroscopic analysis and M. Rajewska (Department of Biotechnology, Intercollegiate Faculty of Biotechnology, University of Gdansk and Medical University of Gdansk, Poland) for her comments on the manuscript and her editorial work.

Open Access This article is distributed under the terms of the Creative Commons Attribution License which permits any use, distribution, and reproduction in any medium, provided the original author(s) and the source are credited.

\section{References}

Angelova, Z., Georgiev, S., \& Roos, W. (2006). Elicitation of plants. Biotechnology \& Biotechnological Equipment, 20, 72-83.

Blilou, I., Ocampo, J. A., \& García-Garrido, J. M. (1999). Resistance of pea roots to endomycorrhizal fungus or Rhizobium correlates with enhanced levels of endogenous salicylic acid. Journal of Experimental Botany, 50, 16631668.

Czajkowski, R., Grabe, G. J., \& van der Wolf, J. M. (2009). Distribution of Dickeya spp. and Pectobacterium carotovorum subsp. carotovorum in naturally infected seed potatoes. European Journal of Plant Pathology, 125, 263-275.
Czajkowski, R., De Boer, W. J., Velvis, H., \& van der Wolf, J. (2010). Systemic colonization of potato plants by soil-borne, GFP-tagged strain of Dickeya sp. biovar 3. Phytopathology, 100, 134-142.

Czajkowski, R., de Boer, W. J., van der Zouwen, P. S., et al. (2012a). Virulence of 'Dickeya solani' and Dickeya dianthicola biovar-1 and -7 strains on potato (Solanum tuberosum). Plant Pathology, 62, 597-610.

Czajkowski, R., Pérombelon, M. C. M., van Veen, J. A., \& van der Wolf, J. M. (2012b). Control of blackleg and tuber soft rot of potato caused by Pectobacterium and Dickeya species: a review. Plant Pathology, 60, 999-1013.

Delaney, T. P., Uknes, S., Vernooij, B., et al. (1994). A central role of salicylic acid in plant disease resistance. Science, 266, $1247-1250$.

Durrant, W. E., \& Dong, X. (2004). Systemic aquired resistance. Annual Review of Phytopathology, 42, 185-209.

Golan, A., Kerem, Z., Tun, O. M., Luzzatto, T., Lipsky, A., \& Yedidia, I. (2010). Combining flow cytometry and $g f p$ reporter gene for quantitative evaluation of Pectobacterium carotovorum ssp. carotovorum in Ornithogalum dubium plantlets. Journal of Applied Microbiology, 108, 1136-1144.

Gozzo, F. (2003). Systemic acquired resistance in crop protection: from nature to a chemical approach. Journal of Agricultural and Food Chemistry, 51, 4487-503.

Hayat, Q., Hayat, S., Irfan, M., \& Ahmad, A. (2010). Effect of exogenous salicylic acid under changing environment: a review. Environmental and Experimental Botany, 68, 14-25.

Heil, M., \& Bostock, R. M. (2002). Induced systemic resistance (ISR) against pathogens in the context of induced plant defences. Annals of Botany, 89, 503-512.

Hélias, V., Hamon, P., Huchet, E., van der Wolf, J. M., \& Andrivon, D. (2011). Two new effective semiselective crystal violet pectate media for isolation of Pectobacterium and Dickeya. Plant Pathology, 61, 339-345.

Kessmann, H., Staub, T., Hofmann, C., et al. (1994). Induction of systemic acquired disease resistance in plants by chemicals. Annual Review of Phytopathology, 32, 439-459.

Lagonenko, L., Lagonenko, A., \& Evtushenkov, A. (2013). Impact of salicylic acid on biofilm formation by plant pathogenic bacteria. Journal of Biology and Earth Sciences, 3, B176-B181.

Laurila, J., Ahola, V., Lehtinen, A., Joutsjoki, T., Hannukkala, A., Rahkonen, A., \& Pirhonen, M. (2008). Characterization of Dickeya strains isolated from potato and river water samples in Finland. European Journal of Plant Pathology, 122, 213-225.

Luzzatto, T., Golan, A., Yishay, M., Bilkis, I., Ben-ari, J., \& Yedidia, I. (2007a). Priming of antimacrobial phenolics during induced resistance response towards Pectobacterium carotovorum in the ornamental monocot calla lily. Journal of Agricultural and Food Chemistry, 55, 10315-10322.

Luzzatto, T., Yishay, M., Lipsky, A., Ion, A., Belausov, E., \& Yedidia, I. (2007b). Efficient, long-lasting resistance against the soft rot bacterium Pectobacterium carotovorum in calla lily provided by the plant activator methyl jasmonate. Plant Pathology, 56, 692-701.

Luzzatto-Knaan, T., Kerem, Z., Doron-Faigenboim, A., \& Yedidia, I. (2014). Priming of protein expression in the defence responce of Zantedeschia aethiopica to 
Pectobacterium carotovorum. Molecular Plant Pathology, 15, 364-378.

Métraux, J., Nawrath, C., \& Genoud, T. (2002). Systemic acquired resistance. Euphytica, 124, 237-243.

Murashige, T., \& Skoog, F. (1962). A revised medium for rapid growth and bio-assays with tobacco tissue cultures. Physiologia Plantarum, 15, 473-497.

Palva, T. K., Hurtig, M., Saindrenan, P., \& Palva, E. T. (1994). Salicylic acid induced resistance to Erwinia carotovora subsp. carotovora in tobacco. Molecular Plant - Microbe Interactions, 7, 356-363.

Pérombelon, M. C. M. (2002). Potato diseases caused by soft rot Erwinias: an overview of pathogenesis. Plant Pathology, 51, $1-12$.

Perombelon, M. C. M., \& Kelman, A. (1980). Ecology of the soft rot Erwinias. Annual Review of Phytopathology, 18, 361-387.

Pérombelon, M. C. M., \& Salmond, G. P. C. (1995). Bacterial soft rots. Pathogenesis and Host Specificity in Plant Diseases, 1, 1-20.

Pieterse, C. M. J., van Pelt, J. A., Ton, J., et al. (2000). Rhizobacteria-mediated induced systemic resistance (ISR) in Arabidopsis requires sensitivity to jasmonate and ethylene but is not accompanied by an increase in their production. Physiological and Molecular Plant Pathology, 57, 123-134.

Pieterse, C. M. J., van Pelt, J. A., van Wees, S. C. M., et al. (2001). Rhizobacteria-mediated induced systemic resistance: triggering, signalling and expression. European Journal of Plant Pathology, 107, 51-61.

Price, C. T. D., Lee, I. R., \& Gustafson, J. E. (2000). The effects of salicylate on bacteria. The International Journal of Biochemistry \& Cell Biology, 32, 1029-1043.

Raskin, I. (1992). Role of salicylic acid in plants. Annual Review of Plant Physiology and Plant Molecular Biology, 43, 439-463.

Ryals, J., Uknes, S., \& Ward, E. (1994). Systemic aquired resistance. Plant Physiology, 104, 1109-12.

Shoresh, M., Yedidia, I., \& Chet, I. (2005). Involvement of jasmonic acid/ethylene signaling pathway in the systemic resistance induced in cucumber by Trichoderma asperellum T203. Phytopathology, 95, 76-84.

Silverman, P., Seskar, M., Kanter, D., Schweizer, P., Metraux, J. P., \& Raskin, I. (1995). Salicylic acid in rice (biosynthesis, conjugation, and possible role). Plant Physiology, 108, 633-639.

Slawiak, M., van Beckhoven, J. R. C. M., Speksnijder, A. G. C. L., Czajkowski, R., Grabe, G., \& van der Wolf, J. M. (2009). Biochemical and genetical analysis reveal a new clade of biovar 3 Dickeya spp. strains isolated from potato in Europe. European Journal of Plant Pathology, 125, 245-261.

Szpitter, A., Narajczyk, M., Maciag-Dorszynska, M., Wegrzyn, G., Lojkowska, E., \& Krolicka, A. (2014). Effect of Dionaea muscipula extract and plumbagin on maceration of potato tissue by Pectobacterium atrosepticum. Annals of Applied Biology, 164, 404-414.

Toth, I. K., van der Wolf, J. M., Saddler, G., et al. (2011). Dickeya species: an emerging problem for potato production in Europe. Plant Pathology, 60, 385-399.

Tsror, L., Erlich, O., Lebiush, S., et al. (2009). Assessment of recent outbreaks of Dickeya sp. (syn. Erwinia chrysanthemi) slow wilt in potato crops in Israel. European Journal of Plant Pathology, 123, 311-320.

Tsror, L., Erlich, O., Lebiush, S., et al. (2011). First report of potato blackleg caused by a biovar 3 Dickeya sp. in Georgia. New Disease Reports, 23, 1.

Vallad, G. E., \& Goodman, R. M. (2004). Systemic acquired resistance and induced systemic resistance in conventional agriculture. Crop Science, 44, 1920-1934.

van der Wolf, J.M., de Boer, S.H. (2007). Bacterial pathogens of potato. Potato Biology and Biotechnology, 619-641.

van der Wolf, J. M., Nijhuis, E. H., Kowalewska, M. J., et al. (2014). Dickeya solani sp. nov., a pectinolytic plant pathogenic bacterium isolated from potato (Solanum tuberosum). International Journal of Systematic and Evolutionary Microbiology, 64, 768-774.

van Loon, L. C., Bakker, P., \& Pieterse, C. M. J. (1998). Systemic resistance induced by rhizosphere bacteria. Annual Review of Phytopathology, 36, 453-483.

Vidal, S., De León, I. P., Denecke, J., \& Palva, E. T. (1997). Salicylic acid and the plant pathogen Erwinia carotovora induce defense genes via antagonistic pathways. The Plant Journal, 11, 115-123. 